
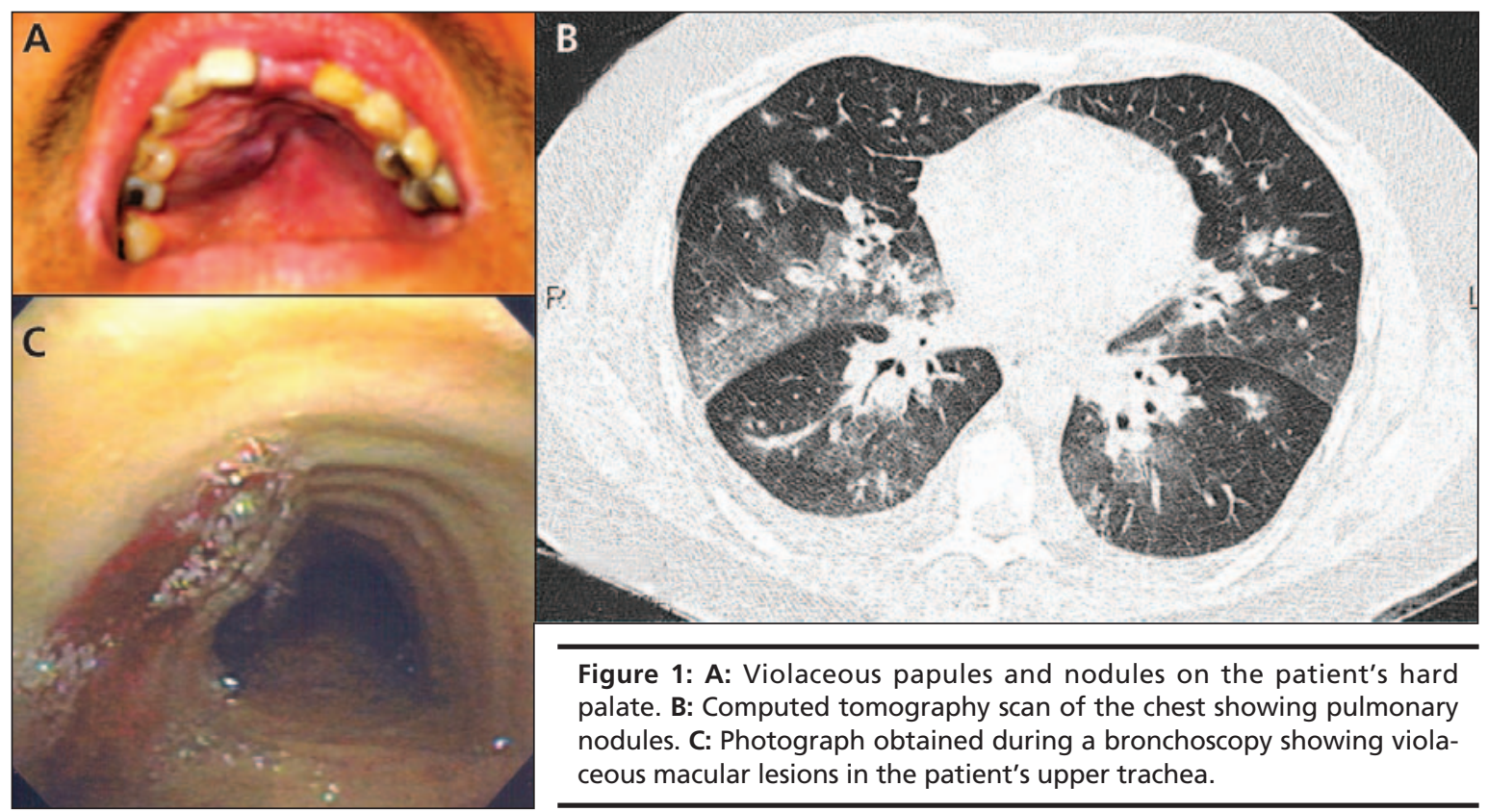

Figure 1: A: Violaceous papules and nodules on the patient's hard palate. B: Computed tomography scan of the chest showing pulmonary nodules. C: Photograph obtained during a bronchoscopy showing violaceous macular lesions in the patient's upper trachea.

\title{
Kaposi sarcoma of the lung
}

A 33-year-old HIV-positive man with a history of immune thrombocytopenia presented with a 1-week history of hemoptysis. He had a Pneumocystis jirovecii pneumonia 1 year before, and he had been receiving highly active antiretroviral therapy for 3 months. Multiple violaceous papules and nodules were visible on his back, chest and hard palate (Figure 1A). The patient's platelet count was 16 (normal 140-400) $\times 10^{9} / \mathrm{L}$, his hemoglobin level was 84 (normal $135-165$ ) g/L, his CD4 count was 66 (normal 500-1400) cells $/ \mathrm{mm}^{3}$ and there was an an undetectable viral load. The results of sputum and blood cultures were negative for acid-fast bacilli and other bacteria. A computed tomography scan of his chest (Figure 1B) showed pulmonary nodules with a peribronchovascular ground-glass pattern and bilateral hilar lymphadenopathy.

A diagnosis of Kaposi sarcoma was confirmed by a positive immunohistochemistry result for HHV-8. Bronchoscopy (Figure 1C) showed scattered violaceous macular lesions in the 5 patient's upper trachea and more confluent lesions throughout the left and main stem bronchi.

The patient was given dexamethasone and a platelet transfusion, and antiretroviral therapy was continued with the goal of raising his CD4 count. Within 2 weeks, his oral and skin lesions had regressed considerably.

Pulmonary involvement occurs in 6\%-32\% of HIV patients who have skin manifestations of Kaposi sarcoma and is associated with a median survival of 19 months and a 5-year overall survival rate of $50 \%$.

Clinical images are medical images chosen because they are particularly intriguing, classic or dramatic. Submissions of clear, appropriately labelled high-resolution images must be accompanied by a figure caption and the patient's written consent for publication. A brief explanation (300 words maximum) of the educational significance of the images with a maximum of 2 references is required.

\section{Christina Kim BSc MD}

Department of Internal Medicine University of British Columbia

Vancouver, BC

David Shu MD

University of British Columbia Department of Medicine Royal Columbian Hospital New Westminster, BC 